\title{
A Cross-Enclave Composition Mechanism for Exascale System Software
}

\author{
Noah Evans \\ Kevin Pedretti \\ Center for Computing Research \\ Sandia National Laboratories \\ \{nevans,ktpedre\}@sandia.gov \\ Michael Lang \\ Ultrascale Systems Research Center \\ Los Alamos National Laboratory \\ mlang@lanl.gov
}

\author{
Brian Kocoloski \\ John Lange \\ Dept. of Computer Science \\ University of Pittsburgh \\ \{briankoco,jacklange\} \\ @cs.pitt.edu \\ Patrick G. Bridges \\ Dept. of Computer Science \\ University of New Mexico \\ bridges@cs.unm.edu
}

\begin{abstract}
As supercomputers move to exascale, the number of cores per node continues to increase, but the I/O bandwidth between nodes is increasing more slowly. This leads to computational power outstripping I/O bandwidth. This growth, in turn, encourages moving as much of an HPC workflow as possible onto the node in order to minimize data movement. One particular method of application composition, enclaves, co-locates different operating systems and runtimes on the same node where they communicate by in situ communication mechanisms.

In this work, we describe a mechanism for communicating between composed applications. We implement a mechanism using Copy on Write cooperating with XEMEM shared memory to provide consistent, implicitly unsynchronized communication across enclaves. We then evaluate this mechanism using a composed application and analytics between the Kitten Lightweight Kernel and Linux on top of the Hobbes Operating System and Runtime. These results show a $3 \%$ overhead compared to an application running in isolation, demonstrating the viability of this approach.
\end{abstract}

\section{INTRODUCTION}

As composed simulation and analytics become a standard part of extreme scale workflows [16,21], making composition accessible to application developers is becoming a necessary feature for future exascale Operating System and Runtime (OS/R) environments. In order to meet the performance and feature needs of each application component, current research efforts are investigating the use of co-kernel architectures in order to provide multiple independent OS/Rs on each local compute node $[12,17,19]$. Running multiple operating systems on the same node makes it possible to

ACM acknowledges that this contribution was authored or co-authored by an employee, or contractor of the national government. As such, the Government retains a nonexclusive, royalty-free right to publish or reproduce this article, or to allow others to do so, for Government purposes only. Permission to make digital or hard copies for personal or classroom use is granted. Copies must bear this notice and the full citation on the first page. Copyrights for components of this work owned by others than ACM must be honored. To copy otherwise, distribute, republish, or post, requires prior specific permission and/or a fee. Request permissions from permissions@acm.org.

ROSS '16, June 1, 2016, Kyoto, Japan

(C) 2016 ACM. ISBN 978-1-4503-4387-9/16/06 . \$ \$15.00

DOI: http://dx.doi.org/10.1145/2931088.2931094 run simulations in bespoke environments that ensure optimal performance while allowing analytics to take advantage of the richer APIs and timesharing features of full weight operating systems. However, this inter-OS composition makes constructing application workflows not just a matter of interprocess communication, it requires new inter-OS communication methods as well.

In this paper we introduce Cross-Enclave Asynchronous Shared Memory (XASM), an approach to composing separate application components using a versioning scheme based on copy-on-write memory semantics. XASM is designed as a composition mechanism for the Hobbes OS/R environment [12]. The Hobbes framework consists of both the Pisces co-kernel architecture [15] as well as XEMEM, a cross OS/R shared memory interface [11]. XEMEM allows applications to setup shared memory regions and route asynchronous notifications between applications running in independent OS/R instances, however it does not provide any higher level communication primitives. XASM is a higher level API that provides a producer-consumer based framework for transferring versioned state information between a simulation and an analytics application.

XASM was designed around the following goals:

1. Provide implicitly synchronized snapshots between operating systems and processes.

2. Preserve performance isolation between the composed operating systems and their applications.

3. Use standard OS APIs and facilities as much as possible in order to minimize the implementation complexity and unfamiliarity when adding composition to existing simulations and analytics frameworks.

Cross-Enclave Asynchronous Shared Memory (XASM) is made possible by using copy on write and custom memory allocators on top of the XEMEM [11] shared memory transport in order to provide cross enclave communication between simulations running on lightweight kernels and analytics running in Linux. The Kitten implementation shares memory at the address space level allowing the use of Kitten's SMARTMAP [4] facilities to share data between processes as well as using XEMEM to share memory across 


\section{Producer Consumer}

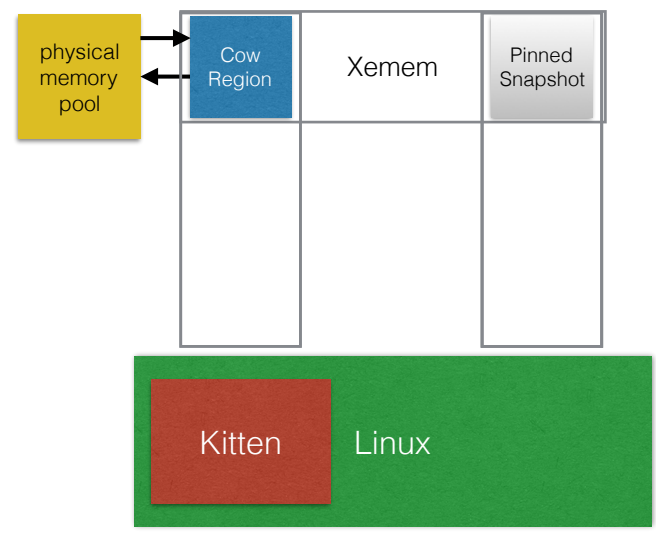

Figure 1: Framework structure: the Copy on Write layer provides explicit synchronization between application and XEMEM provides cross enclave shared memory

enclaves. This allows simulation writers to use preexisting SMARTMAP code with minimal modifications.

XASM makes the following contributions:

- We present XASM, a new mechanism for sharing memory snapshots between co-located virtual machines. The mechanism uses a copy on write technique to decouple the exporting virtual machine from the importing virtual machine, allowing both to proceed asynchronously with their own private view of the memory region.

- We evaluate the performance of XASM and find that its snapshotting capability adds little additional overhead compared to inter-VM shared memory without snapshotting. In most cases, the additional overhead is less than 3\% compared to the application running in isolation, which we argue is a small price to pay for increased capability that snapshotting provides.

- We argue that XASM can be used to simplify the construction of multi-component HPC application compositions, demonstrating a use case involving SNAP [22] coupled with analytics. The asynchronous coupling that XASM provides enables the application components to execute simultaneously without burdening application developers with the need to manually copy data and explicitly synchronize between components.

\section{CROSS ENCLAVE COMMUNICATION IN- FRASTRUCTURE}

XASM consists of a Copy on Write (COW) framework built on top of Cross Enclave Shared Memory (XEMEM). These components provide a mechanism for composing scientific applications and that, when used together, allow the components of an HPC workflow to communicate in a way that minimizes implementation complexity and noise while providing modest performance overheads. The goal of our framework is to allow easy retrofitting of composition into modern simulations and to make it possible to easily and portably write tools that consume data from disparate sources.
HPC applications are often highly complex codes that have been developed over decades of work, with careful performance tuning and validation activies surrounding them. Therefore it is imperative to make composition mechanisms that do not require many changes to the application. Likewise, it is imperative that the performance of the application is not compromised by the composition meachanisms, for example by introducing inter-application resource contention or unnecessary data movement - achieving performance isolation is the basis for much of this work.

Our framework consists of coupled components as depicted in Figure 1. The COW layer provides implicit synchronization between the application and the transport, XEMEM provides cross enclave shared memory. These components work in concert to allow cross application and OS communication.

TCASM Transparently Consistent Asynchronous Shared Memory (TCASM) [1] is a shared memory based transport that allows a producer process, typically an HPC application, to share read-only data in situ with an observer process, typically analytics or checkpointing, without needing to rely on manual synchronization between the producing and observing process. TCASM is implemented using copy on write semantics: the producer registers a region of memory corresponding to its data and then exports a snapshot of that memory when it reaches a consistent state. The implementation of TCASM varies according to its host system. In Linux, TCASM is implemented on top of the Linux VMA subsystem. In Kitten, TCASM is implemented as a set of extensions to the aspace system, Kitten's memory management layer.

Kitten Kitten [13] is a special-purpose OS kernel designed to provide a simple, lightweight environment for executing massively parallel HPC applications. Like previous lightweight kernel OSes, such as Catamount [10] and CNK [7], Kitten uses simple resource management policies (e.g., physically contiguous memory layouts) and provides direct user-level access to network hardware (OS bypass). A key design goal of Kitten is to execute the target workload - highly-scalable parallel applications with non-trivial communication and synchronization requirements - with higher performance and more repeatable performance than is possible with general purpose operating systems. Kitten also supports virtualization capabilities through its integration with Palacios.

Pisces Co-Kernel Architecture Pisces [15] is a cokernel architecture designed to allow multiple specialized $\mathrm{OS} / \mathrm{R}$ instances to execute concurrently on the same local node. Pisces enables the decomposition of a node's hardware resources (CPU cores, memory blocks, and I/O devices) into partitions that are fully managed by independent system software stacks, including OS kernels, device drivers, and I/O management layers. Using Pisces, a local compute node can initialize multiple Kitten OS instances as co-kernel enclaves executing alongside an unmodified Linux host OS. Furthermore, by leveraging Palacios support, virtual machine instances can be created on top of these co-kernels as well. Pisces supports the dynamic assignment and revocation of resources between enclaves. Full co-kernel instances may be created and destroyed in response to workload requirements (e.g., application launch and termination), or individual resources may be revoked from or added to running instances. 
XEMEM Cross enclave shared memory is achieved using the XEMEM shared memory transport. XEMEM is a shared memory transport built on top of SGI's XPMEM [20]. While XPMEM shares memory across processes, XEMEM shares memory across enclaves. XEMEM intentionally maintains an interface which is a small superset of XPMEM in order to provide a familiar and well understand interface. The implementation of XEMEM provides page table sharing between enclaves, regions of memory are pinned directly between enclaves and this shared memory is made visible via a global shared memory database which advertises shared memory segments. This global state allows applications to remain ignorant of the topology of individual segments, regions can be referred to via a global ID rather than being forced to have knowledge of which enclave is sharing which memory.

This in turn allows composed applications to be constructed in a decomposed, publish subscribe manner. Scientific applications create and advertise segment IDs while consumer applications such as visualization or analytics subscribe to a particular segment of interest.

Copy on Write Layer With XEMEM, synchronization between producers and consumers in is entirely cooperative and requires manual synchronization via Inter-Processor Interrupts (IPIs). The Copy on Write (COW) layer provides an alternative to this explicit approach, instead using hardware level page protection to provide implicit inter-enclave synchronization. Applications communicate by exporting point-in-time snapshots of their memory, which can be attached to by receiving applications. When an application has reached a stable state and wishes to export a snapshot, application memory is marked copy on write and a new XEMEM region is created and shared in the global database. The creation of snapshots is published in an out-of-band channel such as a Pisces [15] command queue.

The Copy on Write layer is built on the Kitten Lightweight Kernel [14]. However Kitten, as a lightweight kernel, does not provide traditional virtual memory management. To minimize virtual memory interference with applications, Kitten does not provide demand paging. Virtual to physical memory mappings are established when parts of the virtual memory address space are instantiated at process startup or address space manipulations. Before the COW layer was added, an application page fault would cause the Kitten kernel to panic.

To provide COW for Kitten we added a page fault handler to the Kitten kernel. However, instead of providing a global page fault handler and global pool of physical pages, we add COW functionality to Kitten by allowing individual applications to self page [9] from private, per-process backing pools of physical memory. These backing pools are assigned on a per virtual memory address space level using per page assignment policies. These policies determine which physical pages are chosen from the backing pools and when backing pool memory is freed. This allows applications to choose their page fault replacement policies. Applications with static and regular memory access patterns can allocate and deallocate memory in large, multiple page sized units. Applications with random and irregular memory access patterns can allocate and deallocate memory on a per page basis.

\subsection{Interface}

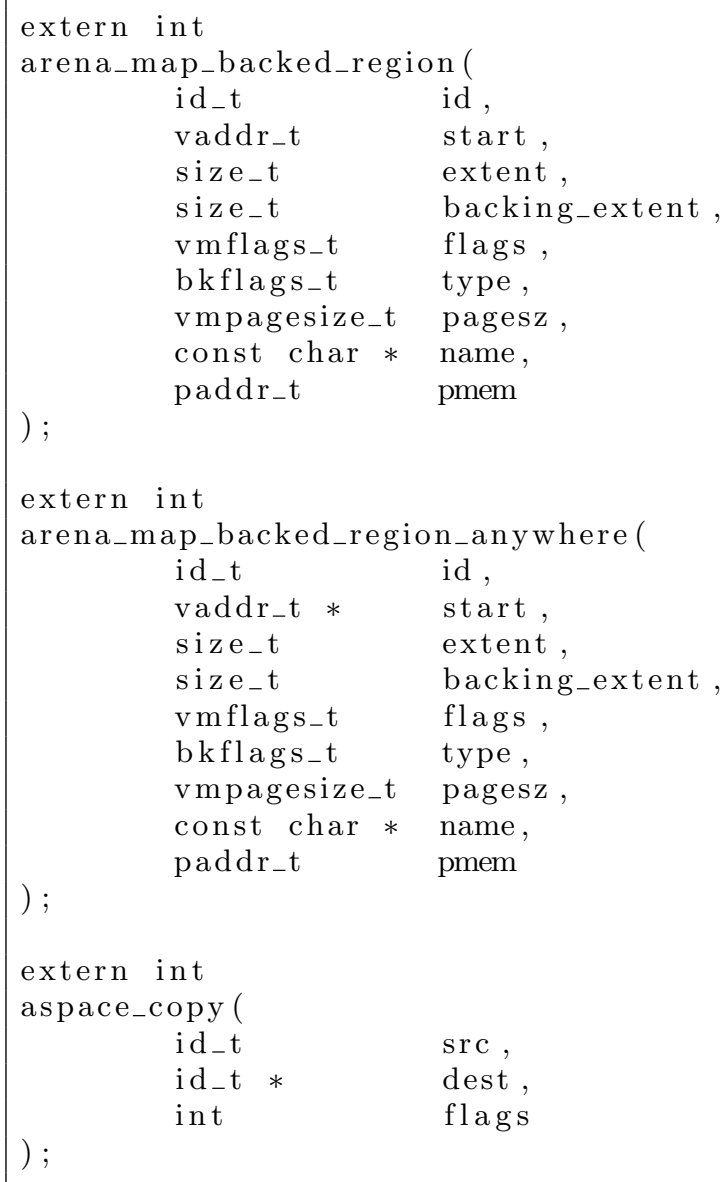

Figure 2: XASM API additions: XASM adds two functions to kitten, arena_map_backed_region and arena_map_backed_region_anywhere allows the user to map in regions of Copy on Write backed memory into an address space. Aspace_copy allows the user to copy address spaces. A side effect of aspace_copy is that the user can snapshot a reified version of the current address space and share it across processes and enclaves. 


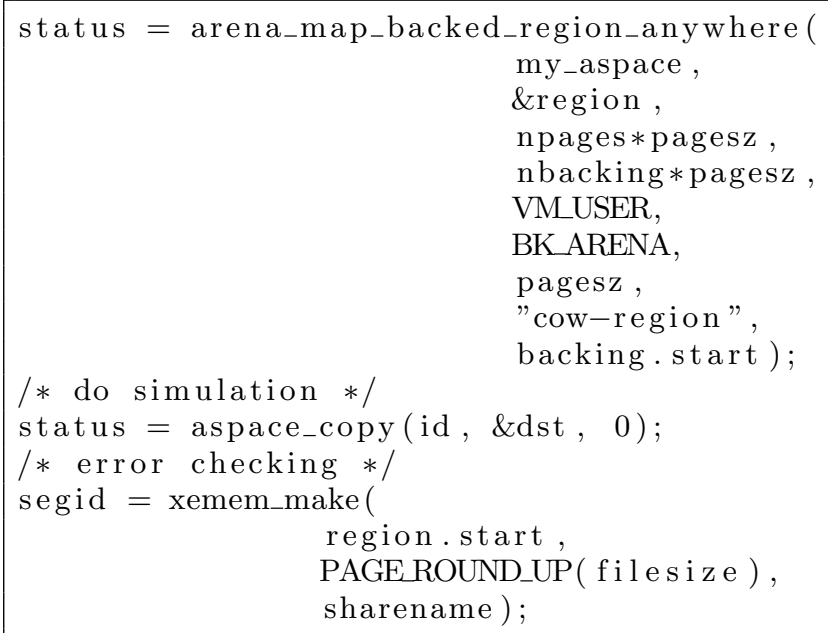

Figure 3: XASM usage: producer. XASM producers create arenas, address spaces backed by per address space physical pages. These address space properties and page tables are reified and copied by aspace_copy before being published by xemem_make

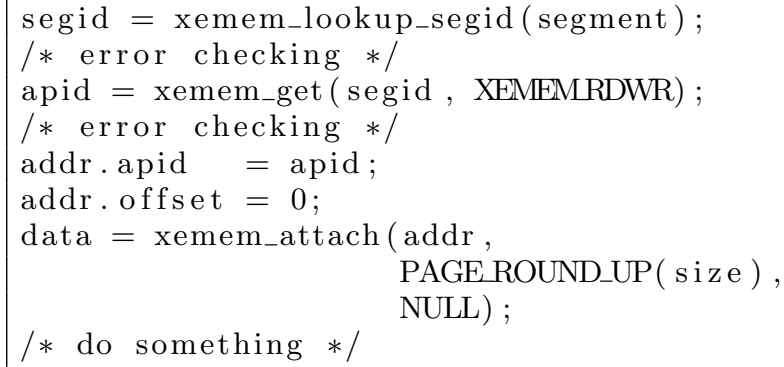

Figure 4: XASM usage: consumer. Consumers of XASM use the traditional XEMEM interface to attach to snapshots. The only difference between XASM and XEMEM is in workflow. XASM creates one new XEMEM segment per snapshot, the protocol for finding new segments is out of band.
The interface to XASM uses the Kitten aspace virtual memory mechanism and SMARTMAP [4] as the basis for sharing COW memory. When users allocate new memory using aspaces, they can use the

arena_map_backed_region_anywhere() call (Figure 2) to allocate a new type of aspace, an arena region of COW backed virtual memory, with backing corresponding to the physical memory page pool used to resolve COW faults.

When the region reaches a stable state the creating application can make an aspace_copy() call to copy the aspace's current state and pagetables from aspace src to dst. An example of this is shown in Figure 3. Since aspace_copy() copies virtual to physical mappings to the new aspace it provides a consistent copy of the virtual to physical mappings at the moment of the copy. This "snapshot" aspace can then be mapped into the address of another local process or shared across enclaves using XEMEM. Figure 4 shows an example of a consumer process attaching to a snapshot.

\section{EXPERIMENTAL SETUP}

We performed our experiments on a dual-socket Intel Xeon E5-2430 clocked at $2.2 \mathrm{GHz}$. Each socket consists of 6 cores, each with a private $32 \mathrm{~KB}$ L1D and $32 \mathrm{~KB}$ L1I caches and a private unified $256 \mathrm{~KB} \mathrm{~L} 2$ cache. Each core features two hardware threads (HyperThreading), and all cores in a socket share a $15 \mathrm{MB}$ unified L3 cache. The system is equipped with $128 \mathrm{~GB}$ of RAM distributed in two NUMA domains. We compiled all applications with gec 4.8.5. Hyperthreading was turned off for these experiments.

\section{PERFORMANCE EVALUATION}

In this section, we use a combination of micro and macro benchmark workloads to demonstrate the performance characteristics of our approach. We begin by quantifying the costs of copy on write on Kitten and Linux, then we measure the costs of XASM using the SNAP [22] neutronics proxy app to test the performance of our approach under real workloads. We refer the reader to [11] for a thorough discussion of the performance implications of XEMEM.

\subsection{Empirical evaluation of Copy on Write and mechanisms}

To understand the performance implications of our approach it is necessary to understand the costs imposed by choosing COW as a sharing mechanism. There are two primary costs to COW handling at the systems level, the cost of handling the page fault interrupt and the cost of page allocation (and potential contention at the virtual memory level) in the kernel.

To measure these costs we instrumented the Linux and Kitten kernels by adding rdtsc instructions to the entry and exit points of page fault handlers in order to measure the costs of page fault handling inside and outside of the kernel.

We then touch COW marked pages in sequence in both systems for 1000 samples on Kitten and 10000 samples on Linux. Figure 5 shows the cost of these COW faults in Linux and Kitten. The Figure 6 clarifies the performance differences between each system.

In the case of Kitten and Linux for the common case we observe that copy on write page fault handling takes approximately 3000 cycles for a fault to be serviced. Kitten's fault handler is more deterministic than Linux's, where Linux ex- 


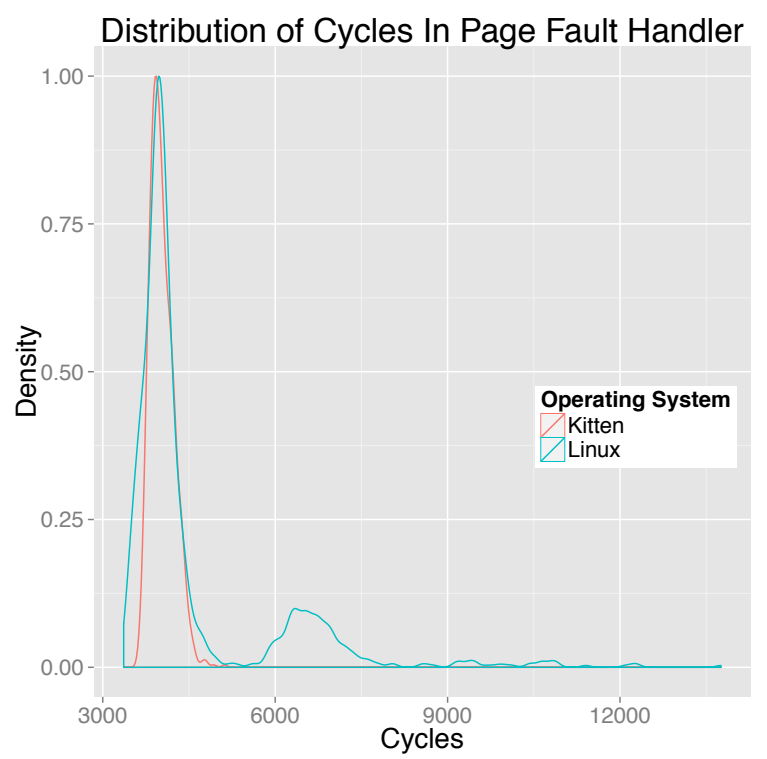

Figure 5: Distribution of pagefault costs of Linux and Kitten. Both operating have similar common case results, but contention for resources in Linux causes occasional delays

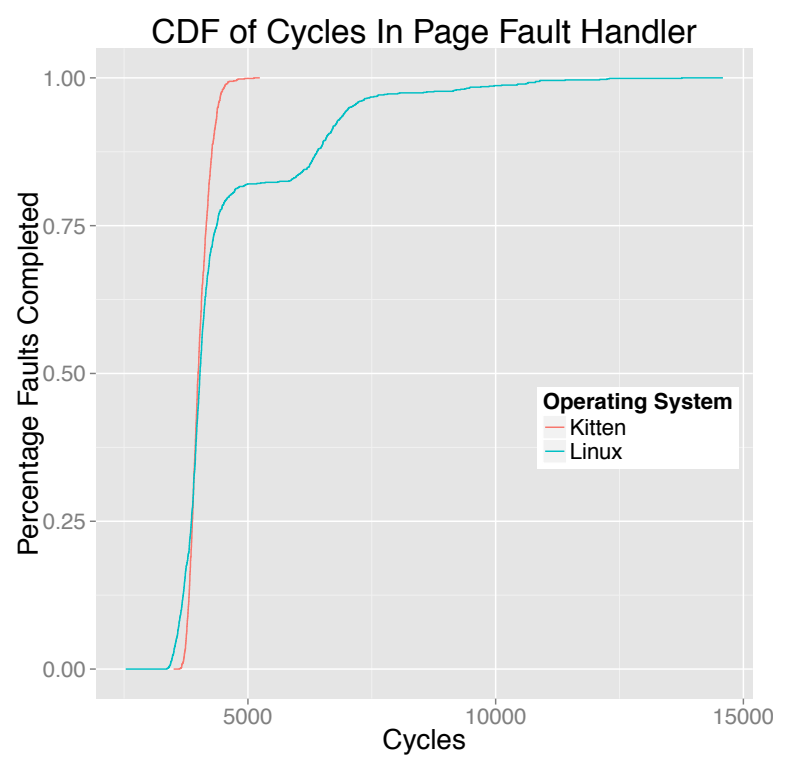

Figure 6: CDF of pagefault costs of Linux and Kitten. We can see that Kitten has extremely regular fault handling performance while Linux suffers from delays $20 \%$ of the time

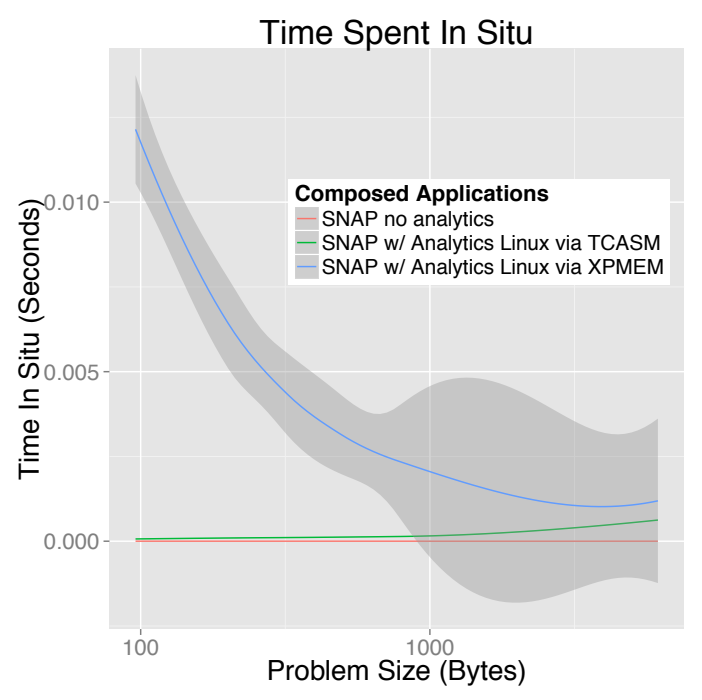

Figure 7: Time SNAP spends in situ in Linux. As the problem size increases the amount of contention between the SNAP and its analytics decreases, meaning that even in the worst case the amount of time spent communicating between workloads decreases. The curve is LOESS fitted and the grey region represents the error in individual measurements.

periences systematic delays of greater than 1000 cycles during approximately $25 \%$ of page faults.

\subsection{Composed SNAP}

Having measured the components of our approach empirically we measure the performance of this approach in a real world application, SNAP [22] coupled with a spectrum analysis analytics workload.

SNAP is a proxy application based on the neutronics transport code PARTISN. PARTISN solves linear Boltzmann transport equations in order to determine the number neutrons and protons in a multi dimensional phase space. SNAP does not actually perform any valid physics, instead mimic the performance requirements and communication of PARTISN. [22]

To model a composed SNAP and analytics workload we couple SNAP with a small spectrum analysis program which runs independently, potentially in a separate enclave. At each timestep SNAP produces a COW snapshot of its internal flux and velocity arrays. The spectrum analysis then reads the data, computes the spectrum of the published data, outputs the result and then waits for the next timestep.

To analyze this composition approach we measure the effect of composition on SNAP in a variety of contexts.

\subsubsection{Linux}

Figure 7 establishes three performance baselines for our framework using Linux. It creates no-op stub functions (e.g., functions which do no work and return) for the composition API in our composed applications to find a lower bound for performance. We then try a composed COW approach to composition on Linux using TCASM [1]. Finally we test measure against a "worst case" that uses spinlocks with no 


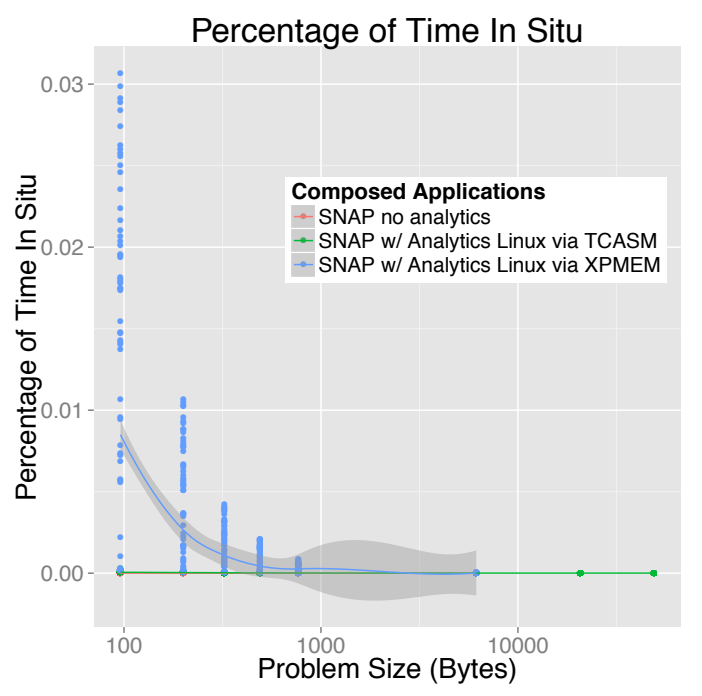

Figure 8: Percentage of time SNAP spent in situ compared to application runtime in Linux (y-axis 0.03 means $3 \%$ ). As the problem size increases SNAP the amount of data shared by SNAP does not increase linearly with the amount of time spent in computation, eventually approaching $0 \%$. The curve is LOESS fitted and the grey region represents the error in individual measurements.

backoff to serialize the the computation and data exchange in SNAP and its analytics over an XPMEM [20].

The results of these measurements show that as SNAP performs non trivial workloads the cost of on-node analytics, baseline, worst-case and COW quickly converge making the composition mechanism irrelevant to the performance of the application. Figure 8 clarifies this assertion not only is the time spent in analytics negligible it is a fraction of a percent of the runtime of the application itself.

\subsubsection{Kitten}

Having established a baseline using Linux, we now test our approach using a Kitten to Linux workload composed between enclaves in order to simulate a real composed application. To do this we use the Hobbes Node Virtualization Layer [3], specifically Pisces [15] co-kernels to create enclaves and communicate across these enclaves using the mechanism described in Section 2.

The results of these measurements show greater but still relatively small overheads. Figure 9 shows the distribution of fault timings for Kitten to Linux composition as the problem size increases. This shows a constant value.

Much of this overhead is explained by the implementation of Kitten's virtual memory system. Kitten does not deal with memory at page granularity instead, to facilitate large pages and RDMA which require large contiguous spans of memory, Kitten allocates memory at the region (segment) level. To share memory across processes Kitten allows the sharing of entire page tables between [4]. This means to publish a snapshot at page table granularity kitten must copy the entire page table to make a snapshot rather than just sharing a particular region of virtual to physical mappings (as Linux does with VMAs). Thus the publishing mech-

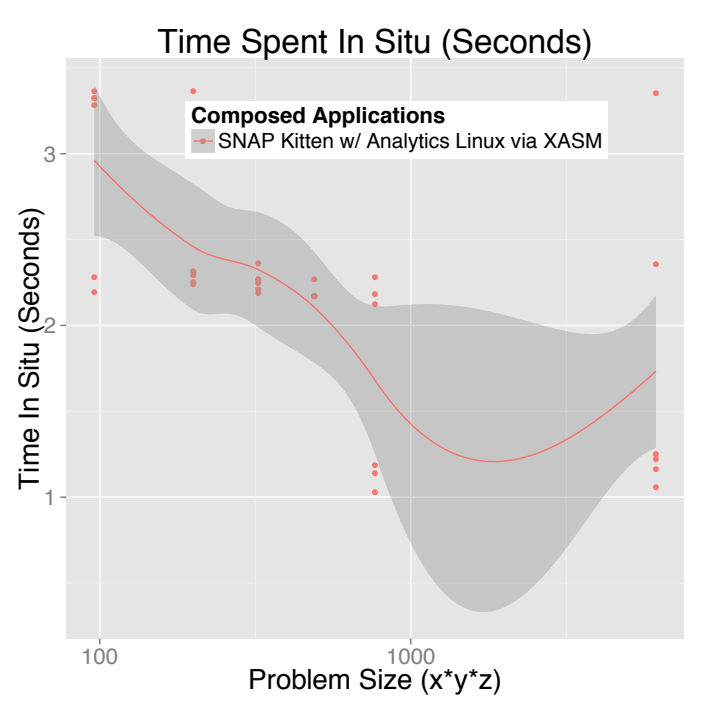

Figure 9: Time SNAP spends in situ in Kitten. Kitten shares data on a per address space basis, necessitating a copy page table copy every time data is shared between SNAP and its analytics. However this time remains relatively constant between runs even as the problem size scales. The curve is LOESS fitted and the grey region represents the error in individual measurements.

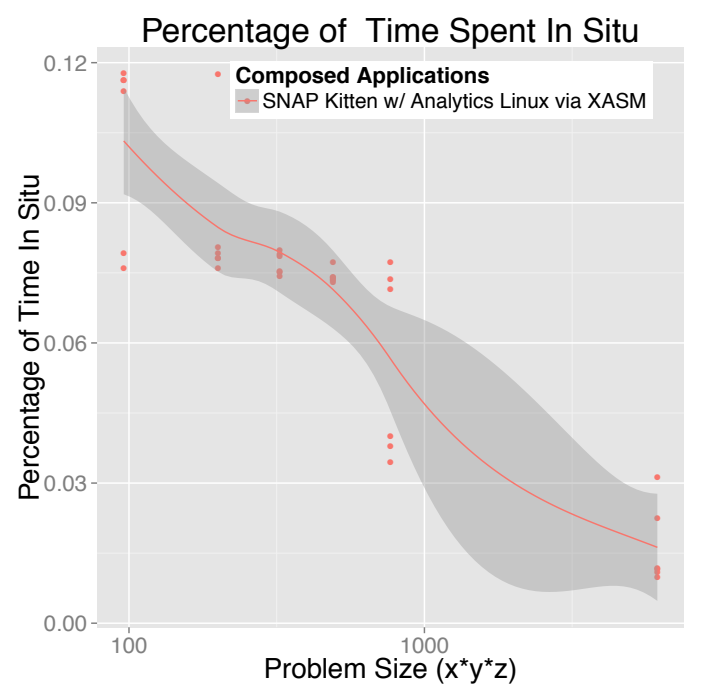

Figure 10: Percentage of time SNAP spent in situ compared to application runtime in Kitten (y-axis .12 means $12 \%)$. As the problem size increases the amount of time spent handling page table copies as a percentage of overall execution time decreases approaching $3 \%$ of runtime or less. The curve is LOESS fitted and the grey region represents the error in individual measurements. 
anism of Kitten snapshotting is more costly compared to Linux.

However, even with these overheads as the problem scales the overheads reduce to $3 \%$ of the total runtime of the application with further optimization possible and remain relatively deterministic. Figure 10 demonstrates this.

\section{RELATED WORK}

Application composition for HPC systems has been a subject of study for more than a decade, as dealing with computation to I/O bandwidth imbalances has become an increasingly important problem at scale. In addition to work done on HPC composition, there has been significant work on inter-Virtual Machine composition and transport mechanisms which we address in this section.

Middleware level approaches

The ADIOS I/O system [2] is a data staging, transport and scheduling solution optimized for HPC systems. ADIOS automatically translates and reformats data for transmission between applications before choosing a transport plugin to transmit data between composed applications. XASM could be used as a transport layer of the ADIOS model, as it makes no attempts to reformat data or do flow control between applications.

Flexpath [6] provides a data reformatting and publish/subscribe model for communication between composed applications. Flexpath is closer to XASM's level of abstraction but still provides features like data reformatting and protocol based publish subscribe that are higher level than XASM, which operates a system level shared memory and synchronization primitive.

Cross VM shared memory Shared memory across virtual machines $[5,18,23]$ has been a well studied problem. VM based mechanisms attempt to create shared memory channels between VMs that do not require reimplementation of traditional application IPC mechanisms across VMs. In order to enable this transparency most VM shared memory mechanisms are implemented at the socket or network level. Making them semantically close to XASM which operates at the traditional OS Shared Memory IPC level, but very different syntactically and at the API level -Xen based shared memory operates at the socket level, XASM operates using traditional shared memory primitives.

HPC Shared Memory In the HPC realm shared memory memory between processes has traditionally been done as a form of MPI acceleration. KNEM [8] and XPMEM [20] originally implemented shared memory transports as ways of accelerating MPI applications. As an extension to the XPMEM model, Leviathan [11] provides a method of cross enclave composition using a shared memory database and pinned pages between enclaves similar to XPMEM but that provides a global namespace that makes it possible for other enclaves to create and find globally visible shared memory regions that can be accessed by any application on the node. Leviathan also provides an Inter-Processor Interrupt (IPI) based signaling mechanism for communication.

XASM is an extension of XEMEM, that uses COW as a mechanism for making the previously explicit signaling and synchronization via interrupt in XEMEM implicit by dealing with concurrency at the hardware rather than user level. Instead of relying on IPI based communication, interrupts are caused implicitly through the operating systems underlying virtual memory subsystem.

\section{CONCLUSION \& FUTURE WORK}

To explore the design space of composed HPC applications, we designed, implemented, and evaluated a Copy on Write mechanism for inter-enclave enclave communication without explicit synchronization. This approach allows HPC application developers to enable composition in applications with a minimum of effort and little additional performance overhead.

\section{Acknowledgments}

This work was supported in part by the 2013 Exascale Operating and Runtime Systems Program from the DOE Office of Science, Advanced Scientific Computing Research, under award number DE-SC0005050, and program manager Sonia Sachs. Sandia National Laboratories is a multi-program laboratory managed and operated by Sandia Corporation, a wholly owned subsidiary of Lockheed Martin Corporation, for the U.S. Department of Energy's National Nuclear Security Administration under contract DE-AC04-94AL85000.

Los Alamos National Laboratory is operated by Los Alamos National Security LLC for the U.S. Department of Energy under contract DE-AC52-06NA25396.

\section{REFERENCES}

[1] H. Akkan, L. Ionkov, and M. Lang. Transparently consistent asynchronous shared memory. In Proceedings of the 3rd International Workshop on Runtime and Operating Systems for Supercomputers, page 6. ACM, 2013.

[2] D. A. Boyuka, S. Lakshminarasimham, X. Zou, Z. Gong, J. Jenkins, E. R. Schendel, N. Podhorszki, Q. Liu, S. Klasky, and N. F. Samatova. Transparent in situ data transformations in adios. In Cluster, Cloud and Grid Computing (CCGrid), 201414 th IEEE/ACM International Symposium on, pages 256-266. IEEE, 2014.

[3] R. Brightwell, R. Oldfield, A. B. Maccabe, and D. E. Bernholdt. Hobbes: Composition and virtualization as the foundations of an extreme-scale OS/R. In Proceedings of the 3rd International Workshop on Runtime and Operating Systems for Supercomputers, page 2. ACM, 2013.

[4] R. Brightwell, K. Pedretti, and T. Hudson. Smartmap: operating system support for efficient data sharing among processes on a multi-core processor. In Proceedings of the 2008 ACM/IEEE conference on Supercomputing, page 25. IEEE Press, 2008.

[5] A. Burtsev, K. Srinivasan, P. Radhakrishnan, K. Voruganti, and G. R. Goodson. Fido: Fast inter-virtual-machine communication for enterprise appliances. In USENIX Annual technical conference. San Diego, CA, 2009.

[6] J. Dayal, D. Bratcher, G. Eisenhauer, K. Schwan, M. Wolf, X. Zhang, H. Abbasi, S. Klasky, and N. Podhorszki. Flexpath: Type-based publish/subscribe system for large-scale science analytics. In Cluster, Cloud and Grid Computing (CCGrid), 2014 14th IEEE/ACM International Symposium on, pages 246-255. IEEE, 2014.

[7] M. Giampapa, T. Gooding, T. Inglett, and R. Wisniewski. Experiences with a Lightweight 
Supercomputer Kernel: Lessons Learned from Blue Gene's CNK. In Proceedings of the 23rd International Conference for High Performance Computing, Networking, Storage and Analysis (SC), 2010.

[8] B. Goglin and S. Moreaud. Knem: A generic and scalable kernel-assisted intra-node mpi communication framework. Journal of Parallel and Distributed Computing, 73(2):176-188, 2013.

[9] S. M. Hand. Self-paging in the nemesis operating system. In OSDI, volume 99, pages 73-86, 1999.

[10] S. M. Kelly, J. P. V. Dyke, and C. T. Vaughan. Catamount N-Way (CNW): An implementation of the Catamount light weight kernel supporting N-cores version 2.0. Technical Report SAND2008-4039P, Sandia National Laboratories, June 2008.

[11] B. Kocoloski and J. Lange. Xemem: Efficient shared memory for composed applications on multi-os $/ \mathrm{r}$ exascale systems. In Proceedings of the 24th International Symposium on High-Performance Parallel and Distributed Computing, pages 89-100. ACM, 2015.

[12] B. Kocoloski, J. Lange, H. Abbasi, D. Bernholdt, T. Jones, J. Dayal, N. Evans, M. Lang, J. Lofstead, K. Pedretti, and P. Bridges. System-level support for composition of applications. In Proc. 5th International Workshop on Runtime and Operating Systems for Supercomputers (ROSS), 2015.

[13] J. Lange, K. Pedretti, T. Hudson, P. Dinda, Z. Cui, L. Xia, P. Bridges, A. Gocke, S. Jaconette, M. Levenhagen, and R. Brightwell. Palacios and Kitten: New High Performance Operating Systems for Scalable Virtualized and Native Supercomputing. In Proceedings of the 24th IEEE International Parallel and Distributed Processing Symposium (IPDPS), 2010.

[14] J. Lange, K. Pedretti, T. Hudson, P. Dinda, Z. Cui, L. Xia, P. Bridges, A. Gocke, S. Jaconette, M. Levenhagen, et al. Palacios and kitten: New high performance operating systems for scalable virtualized and native supercomputing. In Parallel $\&$ Distributed Processing (IPDPS), 2010 IEEE International Symposium on, pages 1-12. IEEE, 2010.

[15] J. Ouyang, B. Kocoloski, J. R. Lange, and K. Pedretti. Achieving performance isolation with lightweight co-kernels. In Proceedings of the 24th International Symposium on High-Performance Parallel and Distributed Computing, pages 149-160. ACM, 2015.

[16] C. Sewell, K. Heitmann, H. Finkel, G. Zagaris, S. T. Parete-Koon, P. K. Fasel, A. Pope, N. Frontiere, L.-t. Lo, B. Messer, et al. Large-scale compute-intensive analysis via a combined in-situ and co-scheduling workflow approach. In Proceedings of the International Conference for High Performance Computing, Networking, Storage and Analysis, page 50. ACM, 2015.

[17] H. Tomita, M. Sato, and Y. Ishikawa. Japan overview talk. In Proc. 2nd International Workshop on Big Data and Extreme-scale Computing (BDEC), 2014.

[18] J. Wang, K.-L. Wright, and K. Gopalan. Xenloop: a transparent high performance inter-vm network loopback. In Proceedings of the 17th international symposium on High performance distributed computing, pages 109-118. ACM, 2008.
[19] R. Wisniewski, T. Inglett, P. Keppel, R. Murty, and R. Riesen. mos: An architecture for extreme-scale operating systems. In Proc. 4th International Workshop on Runtime and Operating Systems for Supercomputers (ROSS), 2014.

[20] M. Woodacre, D. Robb, D. Roe, and K. Feind. The sgi® altixtm 3000 global shared-memory architecture. Silicon Graphics, Inc.(2003), 2005.

[21] J. Woodring, M. Petersen, A. Schmeißer, J. Patchett, J. Ahrens, and H. Hagen. In situ eddy analysis in a high-resolution ocean climate model. Visualization and Computer Graphics, IEEE Transactions on, 22(1):857-866, 2016.

[22] J. Zerr and R. Baker. Snap: Sn (discrete ordinates) application proxy - proxy description, 2013.

[23] X. Zhang, S. McIntosh, P. Rohatgi, and J. L. Griffin. Xensocket: A high-throughput interdomain transport for virtual machines. In Middleware 2007, pages 184-203. Springer, 2007. 